\title{
Streamwise and spanwise vortical structure merging inside the wake of an inclined flat plate
}

\author{
Pavel Procházka ${ }^{1, *}$ and Václav Uruba ${ }^{1,2}$ \\ ${ }^{1}$ Department of Fluid Dynamics, The Czech Academy of Sciences, Institute of Thermomechanics, Dolejškova 5, Prague 8 18200, \\ Czech Republic \\ 2 Department of Power System Engineering, Faculty of Mechanical Engineering, University of West Bohemia, Universitní 8, \\ Pilsen 30614, Czech Republic
}

Received: 29 March 2019 / Accepted: 3 November 2019

\begin{abstract}
The aim of this paper is to study the physics related to lift generation on an airfoil. A new hypothesis [1] of physical mechanism of flight relies on existence of streamwise vortical structures above the wing and inside the wake. The vortices origin as a consequence of flow instability inside the boundary layer developed under adverse pressure gradient. These structures are highly dynamical in nature, they change position and size very rapidly. A simple airfoil in the form of a flat plate with moderate angle of attack is considered in the presented research that generates suitable flow at rather low Reynolds number. Stereo PIV time resolved measurement technique is used to capture high-dynamic data in several planes which are located in the wake and are perpendicular to freestream or parallel to the airfoil. The overall image of the flow field dynamics will be created using POD decomposition. Distinct flow patterns with associated kinetic energy are to be described as well as their role in the studied case. Existence of streamwise vorticity is proved, topology and other parameters are estimated in the paper; however related pressures and forces are not evaluated.
\end{abstract}

Keywords: Flow instability / vorticity / wake / PIV / POD

\section{Introduction}

In more than hundred years, we have witnessed very fast development of aeronautics. The ability to fly an apparatus with wings is proved by many experiments and without any doubts. However, classical mathematical fluid mechanics could not give satisfactory explanation of the mystery of gliding flight.

The simple Newton mechanics suggest the origin of the lift of a tilted flat plate by redirecting a horizontal stream of fluid particles, but indicated a disappointingly small value, proportional to the square of the angle of attack. Thus, to Newton theory the flight of birds was inexplicable, and human flight impossible.

D'Alembert followed up in 1752 by formulating his paradox about zero lift and drag of inviscid incompressible irrotational steady flow. This kind of hypothetical potential flow seemed to describe the airflow around a wing since the viscosity of air is very small. To explain flight, d'Alembert's paradox had to be resolved, but nobody could figure out how and it was still an open problem when

\footnotetext{
* e-mail: prochap@it.cas.cz
}

Wright brothers in 1903 showed that human flight in fact was possible in practice, even if mathematically it was impossible. Mathematical fluid mechanics was then saved from complete collapse by the mathematicians Kutta and Zhukovsky, who explained lift as a result of perturbing potential flow by a large-scale circulating flow or circulation around the $2 \mathrm{D}$ section of a wing. The other physicist Prandtl explained drag as a result of a viscous boundary layer $[1,2]$.

This is the basis of state of the art [3-5], which essentially is a simplistic theory for lift without drag at small angles of attack in inviscid flow and for drag without lift in viscous flow. The theory allows an airplane engineer to roughly compute the lift of a wing at cruising speed at a small angle of attack, but not the drag, and not lift and drag at the critical phase of take-off [5]. The lack of mathematics has to be compensated by experiment and experience.

A possible explanation of force generation in fluid dynamics was presented recently by Hoffman, Jansson and Johnson, mathematicians from KTH Stockholm [6]. They supposed fully 3D turbulent flow around the body. The crucial role in forces generation is attributed to presence of streamwise counter-rotating low-pressure rolls of swirling flow system close to the suction surface of the airfoil. Thus, 
the pressure energy is transformed into kinetic energy of vortical motion and low pressure is generated. This mechanism is able to explain origin of both lift and drag forces and the d'Alembert's paradox is resolved [7].

The main goal of the presented paper is to prove existence of vortical structures at the suction region of an airfoil and to estimate their parameters.

The flow around prismatic profile is commonly considered with $2 \mathrm{D}$ character. This assumption is generally valid only for laminar case. For turbulence the flow is homogenous along the spanwise direction only for timeaveraged quantities (velocity modulus, turbulent kinetic energy (TKE), etc.).

This experimental study is performed for the simplest case of the airfoil - the flat plate with semi-cylindrical edges. During foregoing studies, a detail study of the flow field in the vicinity of such profile was examined. For example, the separation zone behind leading edge and vortex shedding frequency from longitudinal plane [8] or the information about the shear stresses at suction side [9] was presented recently. Unlike the previously mentioned studies, current investigation of vorticity is based on measurement taken by stereo (3C2D) Particle Image Velocimetry (PIV) measurements. More, the wake is examined in two sets of planes almost perpendicular to each other. This strategy enables us to detect streamwise and spanwise vorticity and their interactions. Since the flow has strong dynamic nature, analysis by Proper Orthogonal Decomposition (POD) will be used to detect dynamical flow structures and to assess their contribution on the overall situation.

\section{Experimental setup}

The existing blow-down facility is used for the experimental study with open test section. The model is placed in the potential core of free-jet issuing from the wind tunnel contraction.

\subsection{Model geometry}

The simple airfoil in the form of flat plate with semicylindrical leading and trailing edges was used as a test case. Although this is not a fully-fledged airfoil, it is absolutely sufficient for experimental modeling of studied effect. The angle of attack was set to $7^{\circ}$ with respect to the incoming flow. The freestream is generated by blow-down facility with cross-section dimension $250 \times 250 \mathrm{~mm}^{2}$. The flow is perfectly homogenous with speed equal to $5 \mathrm{~m} \mathrm{~s}^{-1}$ (alternatively $10 \mathrm{~m} \mathrm{~s}^{-1}$ ), low turbulence intensity $0.2 \%$ and velocity deviations up to $1 \%$. The chord length of the plate is $c=100 \mathrm{~mm}$, the thickness is $2 \mathrm{~mm}$ and span dimension is over $450 \mathrm{~mm}$. The corresponding Reynolds number is then $33 \times 10^{3}$ and $66 \times 10^{3}$ for 5 and $10 \mathrm{~ms} \mathrm{~s}^{-1}$, respectively.

The scheme of experimental setup can be seen in Figure 1. The Cartesian coordinate system origin is placed in the middle of the leading edge. The $x$ - and $y$-axis are oriented in the perpendicular planes (green color), the $z$ axis is in the direction of airstream. Several perpendicular planes $(z / c=1,1.3,1.5,1.8,2,2.3$ and 2.5$)$ were examined

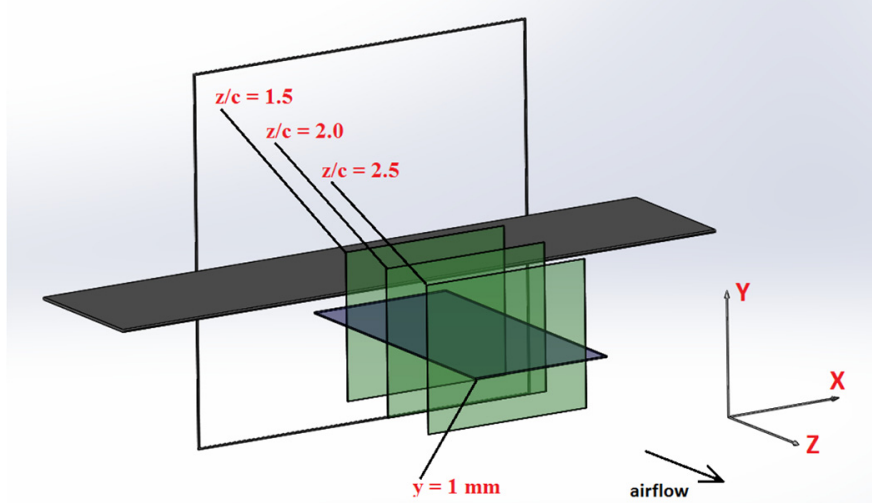

Fig. 1. Setup of rotatable mounted wing and blow-down wind tunnel orifice, green parallel planes perpendicular to flow direction, one plane parallel to suction surface.

to study mainly the streamwise vorticity. The trailing edge is situated in $z / c=1$. All these non-dimension positions of planes were set inside the wake. One plane of measurement was established to cross all previously mentioned which should provide the existence of spanwise vortical structures [8]. This plane (blue color in Fig. 1 and white plane in Fig. 2) is oriented parallel to the flat plate, behind it and $1 \mathrm{~mm}$ above it. The plane is not complementary but necessary to fulfil the information about structure merging.

\subsection{Measurement technique}

Stereo TR-PIV technique enables to measure all three velocity component in the plane of measurement with sufficient high resolution. The measurement device is from Dantec company and it is based on laser with cylindrical optics and two CMOS cameras. The camera NanoSense MKIII has full resolution $1280 \times 1024$ pixels for maximal acquisition frequency 500 double snaps per second. During investigation, half resolution was used which enables to data acquisition with frequency of $1 \mathrm{kHz}$. The laser New Wave Pegasus Nd:YLF has double head emitting green light with wavelength $527 \mathrm{~nm}$, maximal frequency is $10 \mathrm{kHz}$, shot energy is $10 \mathrm{~mJ}$ (for $1 \mathrm{kHz}$ ) and corresponding power is $10 \mathrm{~W}$ per head.

To measure and post-process data, Dynamic Studio ver. 6.2 was utilized. Standard calibration target $100 \times 100 \mathrm{~mm}$ was used. The target was traversed into five distinct positions by stepper motor and Pinhole camera calibration was performed with resulting error less than 0.2 pixels. Perpendicular planes had Field of View (FoV) with dimensions $60 \times 25 \mathrm{~mm}^{2}$ which covered sufficient span of the airfoil as well as the height of the wake. The correlation algorithm evaluated $68 \times 29$ vectors with maximal $10 \%$ of substituted ones. The acquisition frequency was set to $100 \mathrm{~Hz}$ (10 s of record) for statistical quantities evaluation and to $1 \mathrm{kHz}$ for dynamical analysis (time of recording about $2 \mathrm{~s}$ ). One parallel plane was acquired for similar values. Random error of velocity vector modulus is expected to be up to $2 \%$. 


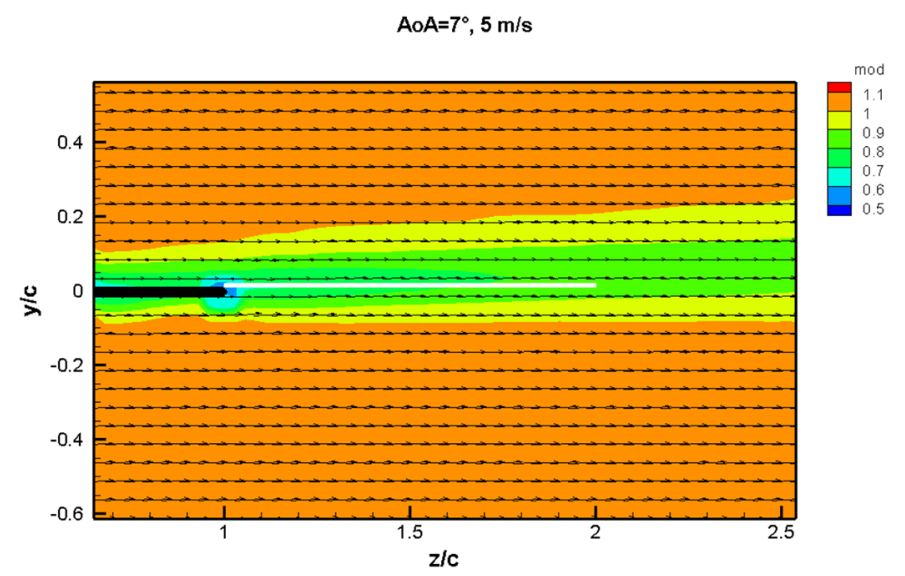

Fig. 2. Time-averaged velocities (normalized) in the wake, the orientation of parallel plane of measurement.

\subsection{Data analysis}

Dynamical flow structures will be identified from the time resolved spatial data using POD method. This method is applied on datasets with two velocity components very often (see e.g. [10]), however in the presented study all three velocity components are taken into account. Thus, the velocity component oriented identically with the vortex core could be detected. The POD analysis can extract the most energetic and dominant coherent structures from turbulent random flow field. The dynamical analysis is performed on velocity fluctuation fields, the time-averaged velocity distribution is not considered in this process, it is analyzed separately. The result of analysis is a set of orthonormal modes ordered according to the relative energy content. The total number of mode corresponds to number of PIV snapshots. Details can be found e.g. in [10]. The low-order modes contain high amount of energy and their topology is created by significant flow structures. On the other hand, high-order modes (low-energy) are created very often from noise (turbulent, error) and can be neglected. The energy fraction contained in a given mode should be considered, low-energy modes are characterized by negligible energy fraction, let say less than $1 \%$ of the total TKE.

Both types of planes will be examined by POD although the resulted POD modes will be interpreted in different ways.

\section{Results}

This section will be devoted to results which were acquired by freestream velocity $5 \mathrm{~m} \mathrm{~s}^{-1}$. The double value of velocity will be introduced only in the case of mode energy comparison. The velocity vector fields will be presented here for both planes of measurement. Generally, black vectors represent the velocity vector projected into the measurement plane ( $x y$ or $x z$ ). The color with color bar represents the distribution of scalar quantities (vel. modulus, vel. component, TKE, vorticity). Green lines were added to make visible the streamlines or for vorticity isolines distribution. The black solid line denotes the

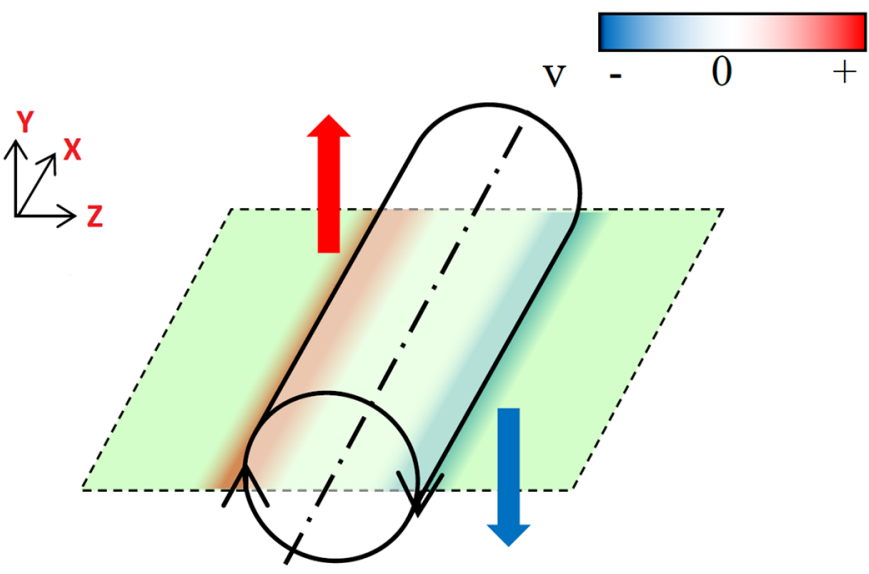

Fig. 3. Scheme of the vortex tube laying in the plane of measurement.

projection (or position) of trailing edge in the direction of $z$-coordinate and black dashed line denoted the projection of trailing edge in the direction of inclined plate (both lines are identical only in position $z / c=1$ ).

All plotted figures are in non-dimensional form. The chord length and inlet velocity was taken as a reference value for coordinates and velocities. The turbulent kinetic energy is related to the mean flow kinetic energy.

The vortical structures visualization from POD modes (and also from inst. snapshots) is performed using streamlines and using vorticity distribution. The vorticity cannot distinguish between circular motion and shear layer but there is no condition for shear layer to be created in this region. There is also another way to indicate presence, shape and orientation of the vortex structures laying in the plane of measurement. This strategy is outlined in Figure 3. If the vortex core axis is oriented inside the plane of measurement, the vortex itself is not detectable by vorticity. Then it is possible to detect positive and negative out-of-plane velocity component using stereo PIV. The position of vortex core is to be estimated based on couple of regions with opposite sign (however this strategy does not provide unambiguous result). This method nevertheless gives the reliable information about spanwise vorticity distribution.

Following four sections will be devoted to timeaveraged quantities, instantaneous topology of the flow and POD decomposition. Both types of planes will be examined together.

\subsection{Mean flow field}

Since the used airfoil is prismatic, the topology of the flow field should be $2 \mathrm{D}$ in the case of mean quantities. The comparison of four distinct planes perpendicular to incoming flow can be seen in Figure 4. The topology is close to be homogenous along span dimension (the deviations are caused by PIV method errors, model geometry imperfections, etc.) and no streamwise-oriented vortical structures are possible to detect. The minimal wake velocities is just behind the trailing edge in $z / c=1$ (not shown here) and this area of local minima is shifted 
Mean flow field, $z / c=1.3$

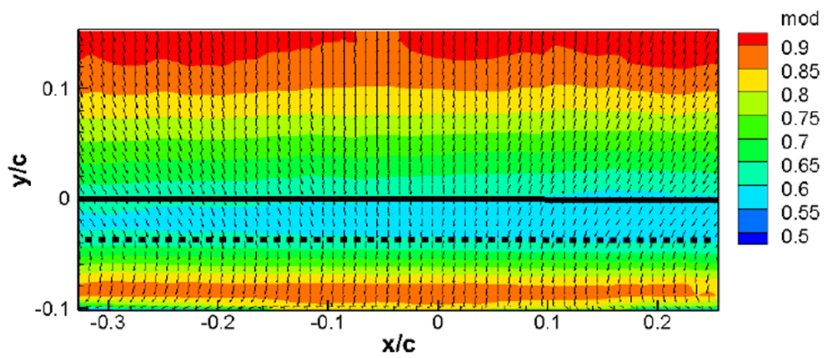

(a)

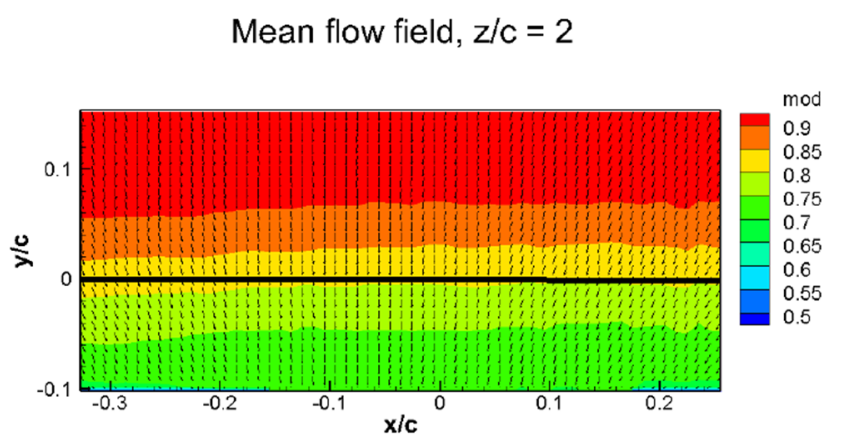

(c)
Mean flow field, $z / c=1.5$

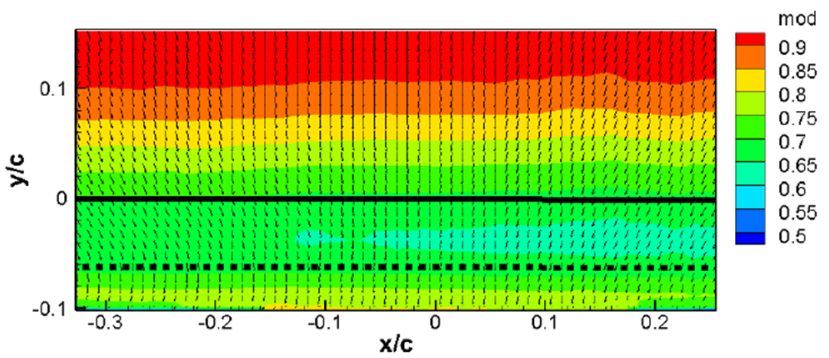

(b)

Mean flow field, $z / c=2.5$

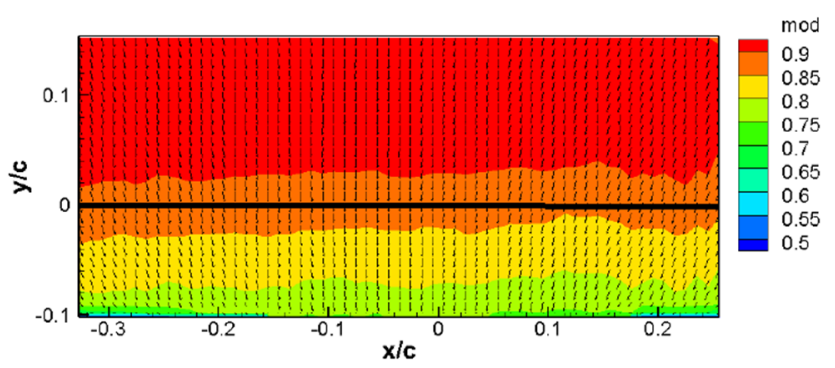

(d)

Fig. 4. Time-averaged flow field in planes $z / c=1.3$ (a), 1.5 (b), 2 (c) and 2.5 (d), distribution of velocity modulus.

below for another planes in downstream direction (this area is located between solid and dashed lines each time). For the last plane $z / c=2.5$, the wake center is out of FoV. The downwash effect can be seen from the arrow orientation.

The distribution of in-plane and out-of-plane velocity component of the parallel plane of measurement is visible in Figure 5. The $u$-velocity component (along span direction) is close to zero everywhere. The value of $v$ is rather small (up to 7\%) of the inlet velocity with local maxima close to the trailing edge position where the downwash is the most obvious. The streamwise velocity component $w$ represents the distribution along the wake. Again, it can be said that the distribution is almost homogenous along $x$-coordinate.

TKE is given here to represent the fluctuation activity of the wake. The distribution for both planes can be seen from Figures 6 and 7. The perpendicular planes revealed that maximal variation should be expected above the wing and in the upper part of the wake. The parallel plane shows the maximal fluctuation just behind the trailing edge with rapid decrease in downstream direction.

\subsection{Instantaneous velocities}

It can be clearly seen from PIV snapshots (Figs. 8 and 9) that instantaneous velocity vector field is completely different than the mean flow. The FoV is full of various vortices, the flow is irregular and perturbed. The wake is extended with downstream coordinate and due to downwash effect is deflected below (see also [8]). While the velocity outside the wake is close to inlet velocity $5 \mathrm{~m} \mathrm{~s}^{1}$ (red color), the wake center is full of streamwise-oriented vortices accompanied by speed deceleration (left column of
Fig. 8). The wake boundary is changing rapidly in time, the topology of the flow is very dynamic. Flow structures are situated rather in the upper part of the wake as their origin was initiated at the suction side of the airfoil. The flow is not definitely regular in span direction. The vorticity distribution clearly identifies individual vortices which are made more visible using streamlines. There are smaller structures just behind the trailing edge $(z / c=1.3)$, then they merge with each other into larger structure $(z /$ $c=2.5)$. The physical mechanism of the small structures merging is not clear and it is to be studied in future. The downwash effect is present in the whole wake region, although it is accompanied by strong vorticity in the planes closest to the trailing edge.

The parallel plane was examined especially to uncover the spanwise vorticity. Two randomly chosen consecutive flow fields are visible in Figure 9. The color represents the distribution of out-of-plane component. The time step between them is $2 \mathrm{~ms}$. Both snaps show the same flow pattern which has changed only slightly. The shift is approximately 0.08 which corresponds to shift velocity about $4 \mathrm{~ms}^{1}$. The in-plane vectors show wavy pattern, while scalar distribution shows the presence of oblique structures. So the flow is filled not only by streamwise oriented structures (Fig. 8) but also by spanwise structures which are oriented in oblique sense.

The strong dynamical behaviour of flow pattern is obvious. Next part will be devoted to analysis of dynamics. The decomposition was applied to velocity fluctuations which were calculated from the same instantaneous velocity fields. The example of fluctuation of the in-plane component $u^{\prime}$ and $w^{\prime}$ is given in Figure 10. The color 


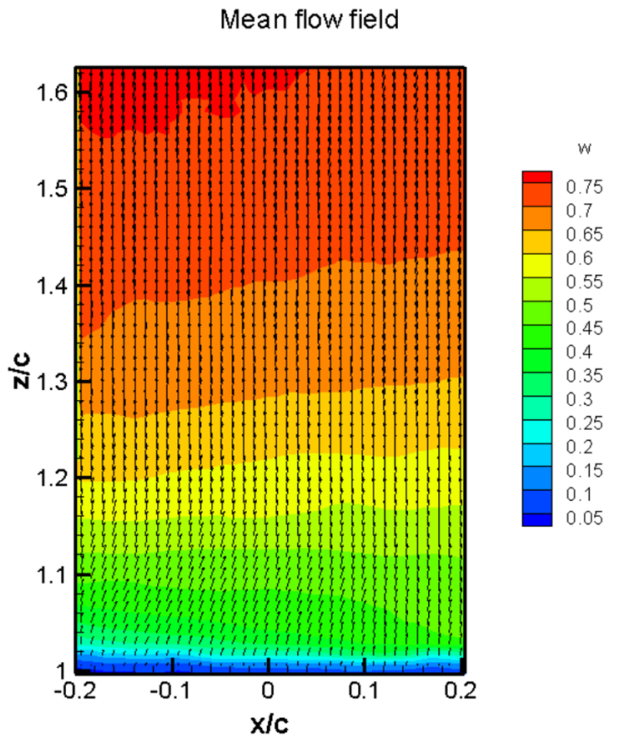

(a)

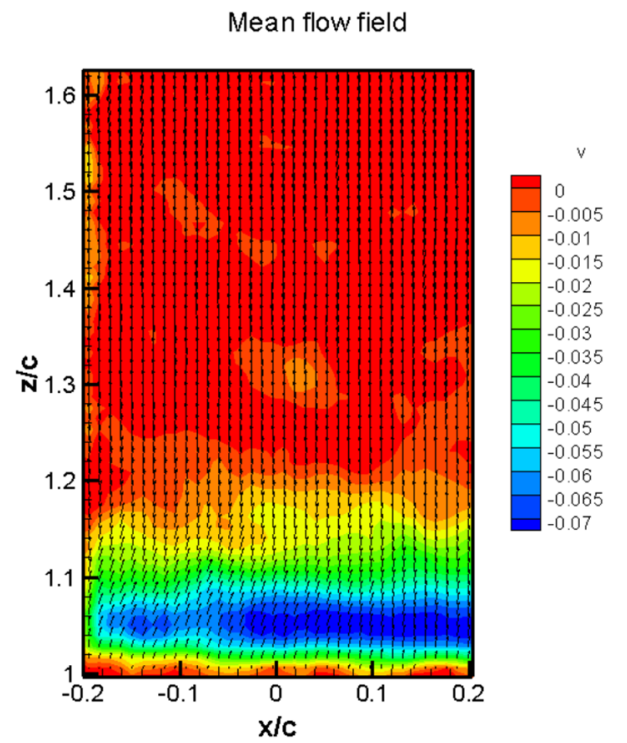

(b)

Fig. 5. Time-averaged flow field for parallel plane in the airfoil wake; distribution of $w$ (a) and $v$-component (b) of velocity.

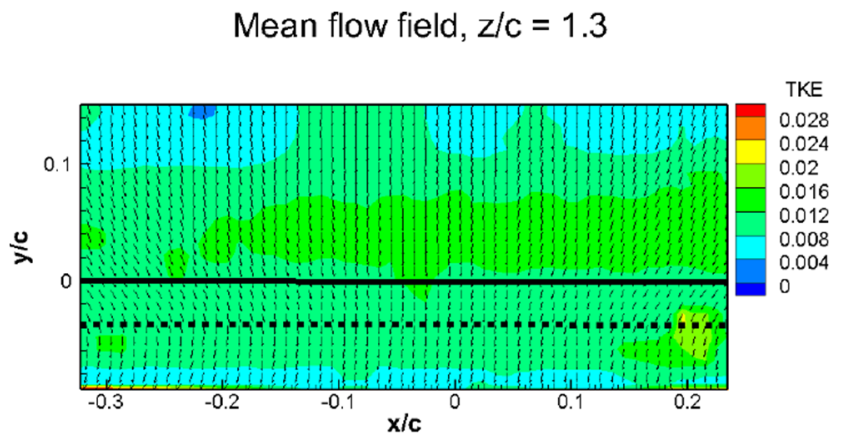

(a)

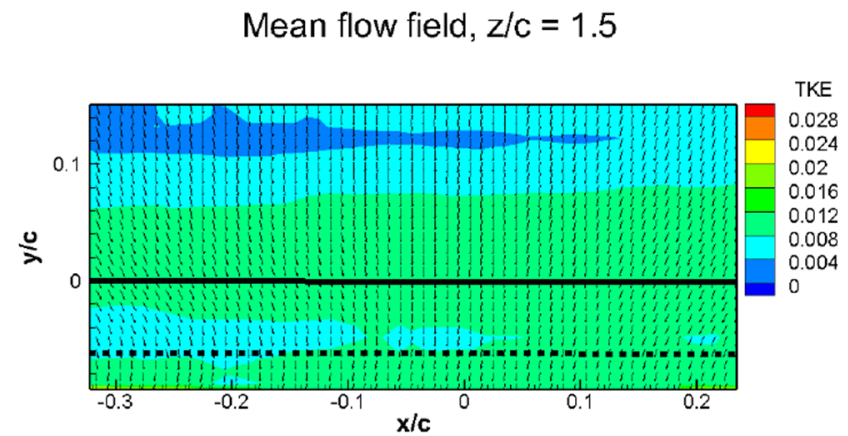

(b)

Fig. 6. Normalized turbulent kinetic energy distribution in planes $z / c=1.3$ (a) and 1.5 (b), fluctuation activity inside the wake.

represents the vorticity $\omega_{\mathrm{y}}$. Both snaps are plotted for the same moments as in Figure 9. An irregular pattern can be seen and it is far from being homogenous and 2D.

\subsection{POD modes}

The topology of dynamic structure is further studied by POD decomposition. This method is detailed described e.g. in [10]. Previously it was mentioned that low-order modes contain the most dominant flow structures concerning kinetic energy content. The POD analysis was performed in all investigated planes and for both inlet velocities. Figures 11 and 12 show the distribution of low-order modes energy in the very first perpendicular plane and in parallel plane, respectively. Only approximately 10 first modes have higher energy than $2 \%$. The very first modes do reach up to $5 \%$ at most. Figure 11 reveals that first POD modes detected under inlet velocity $5 \mathrm{~ms}^{1}$ have higher amount of energy compared to double velocity value which should imply that contain more energetic structures. Contrary, modes with higher amount of energy were

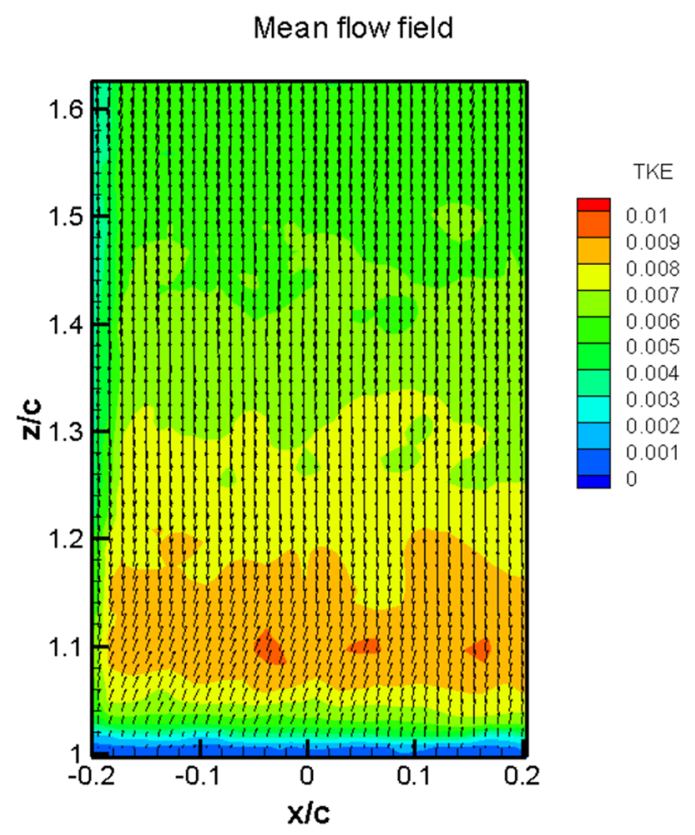

Fig. 7. Normalized TKE distribution in parallel plane. 
Inst. flow field, $z / c=1.3$

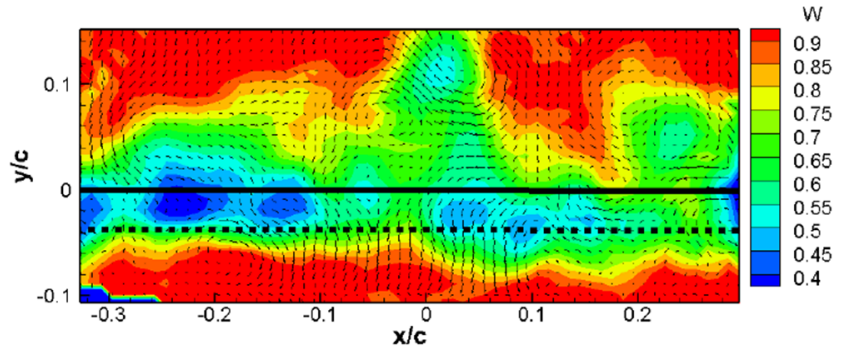

(a)

Inst. flow field, $z / c=1.5$

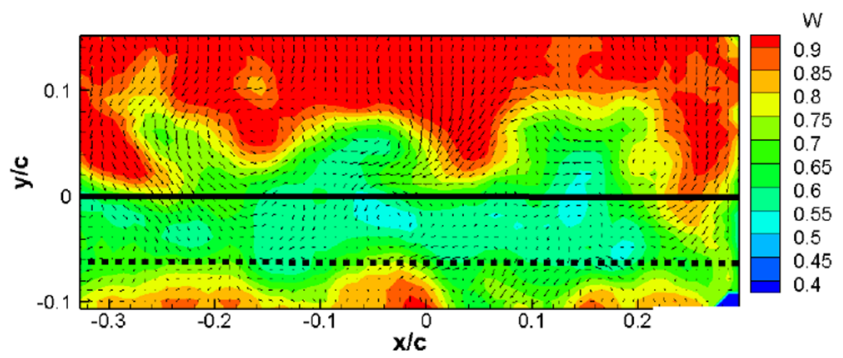

(c)

Inst. flow field, $z / c=2$

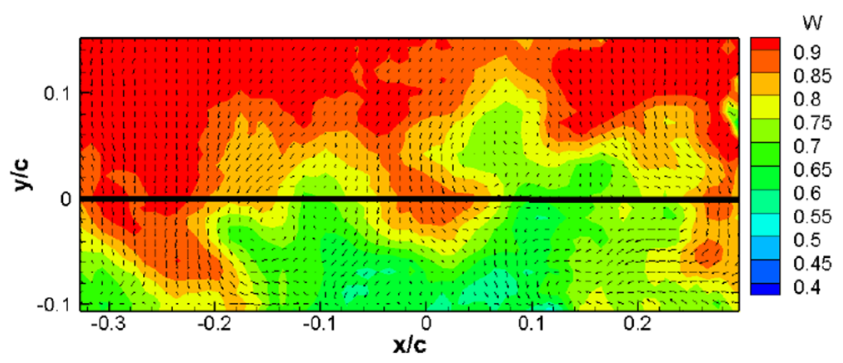

(e)

Inst. flow field, $z / c=2.5$

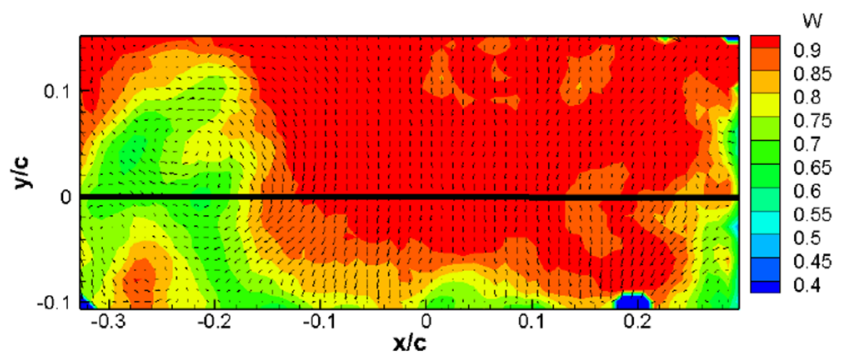

(g)
Inst. flow field, $z / c=1.3$

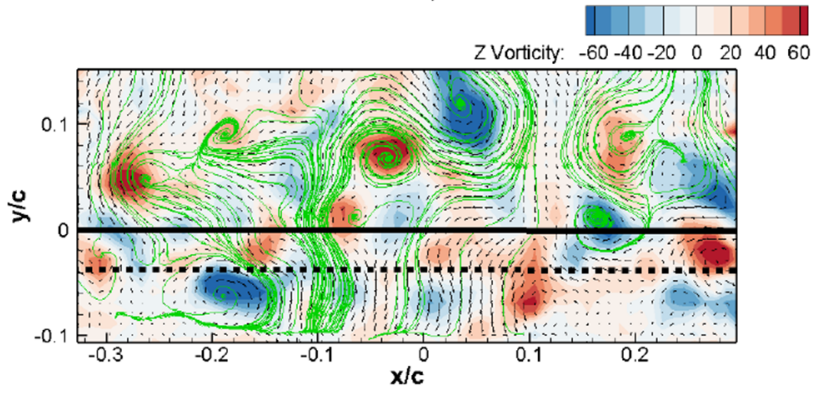

(b)

Inst. flow field, $z / c=1.5$

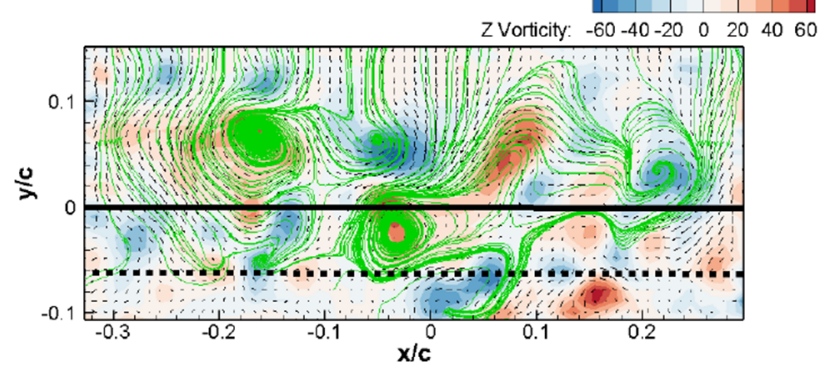

(d)

Inst. flow field, $z / c=2$

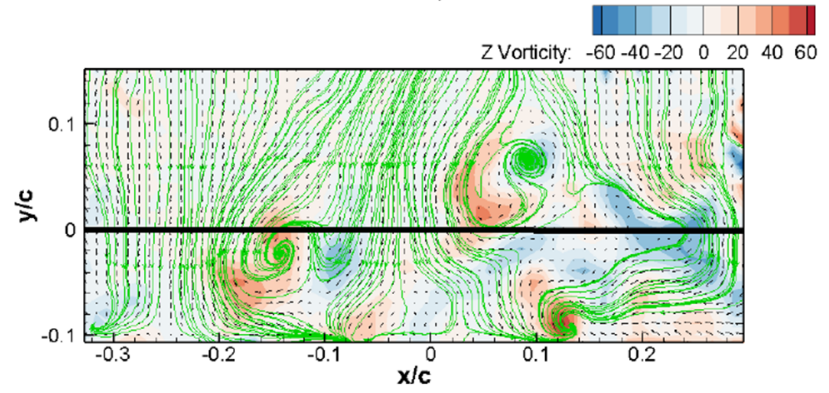

(f)

Inst. flow field, $z / c=2.5$

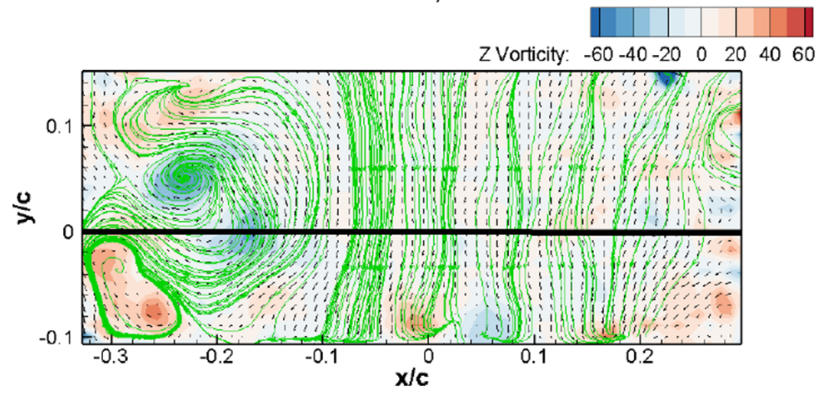

(h)

Fig. 8. Instantaneous velocity vector maps plotted using streamwise velocity component and vorticity distribution with streamlines in planes $z / c=1.3(\mathrm{a}, \mathrm{b}), z / c=1.5(\mathrm{c}, \mathrm{d}), z / c=2(\mathrm{e}, \mathrm{f})$ and $z / c=2.5(\mathrm{~g}, \mathrm{~h})$. 

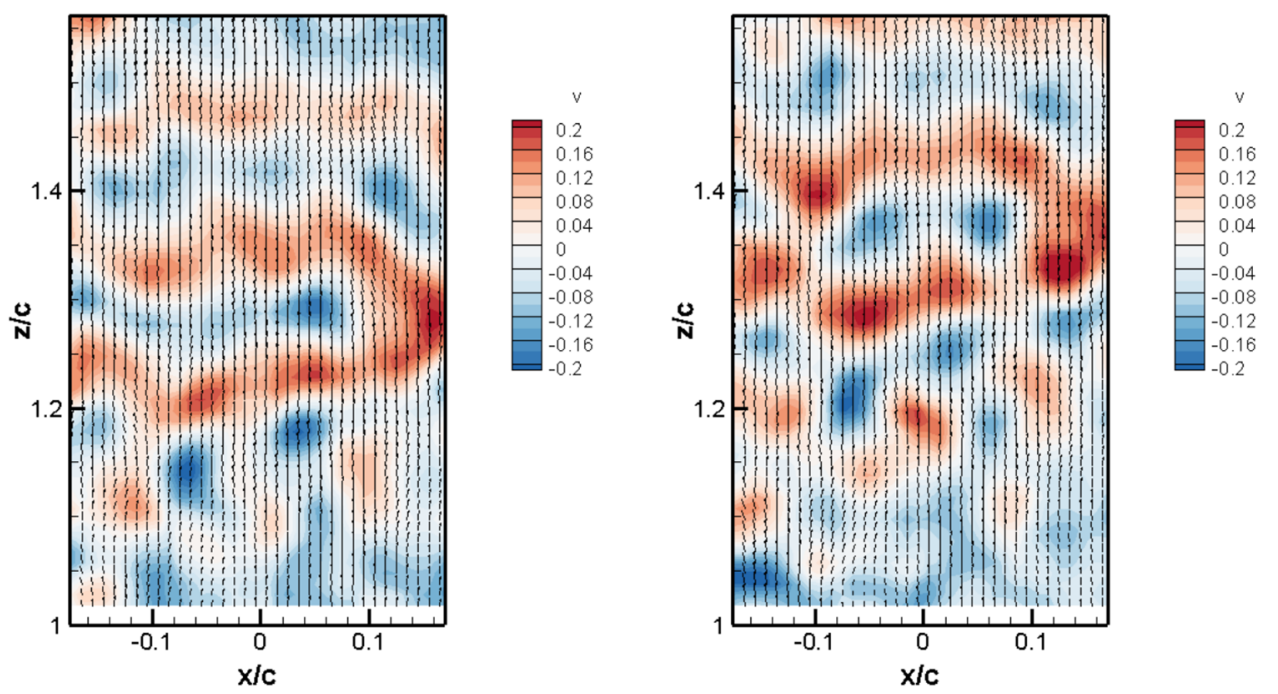

Fig. 9. Two examples of instantaneous flow field, distribution of out-of-plane velocity component.
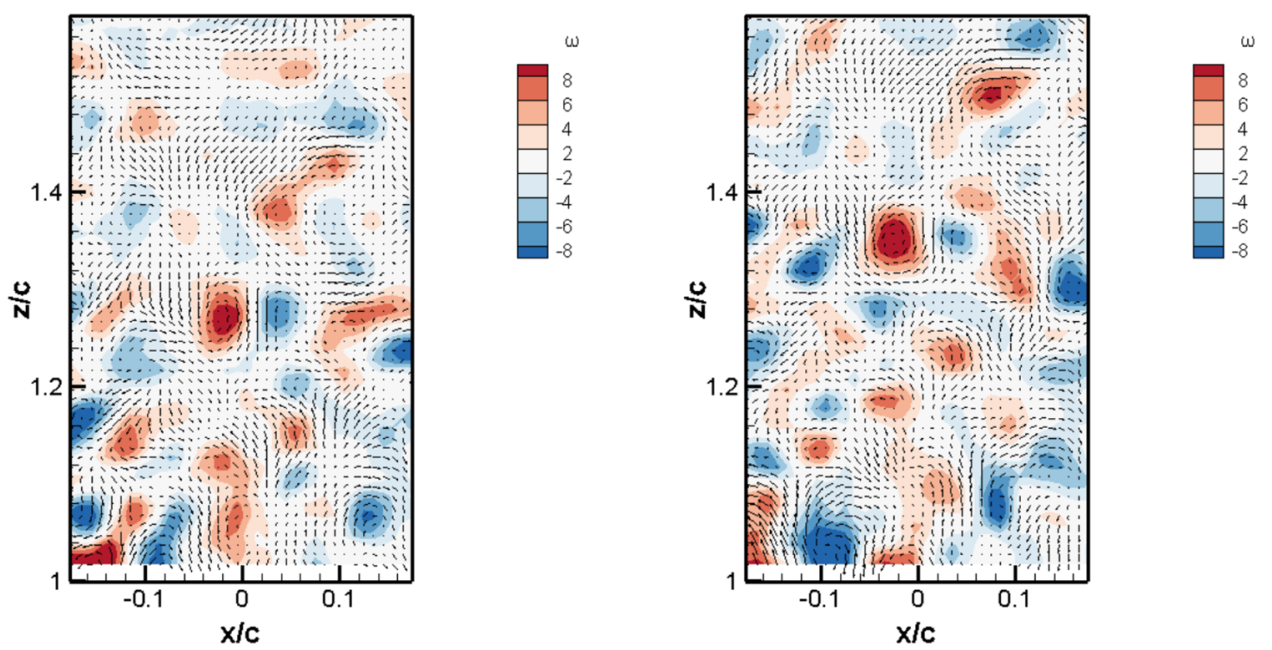

Fig. 10. Two examples of instantaneous in-plane velocity fluctuation, distribution of vorticity.

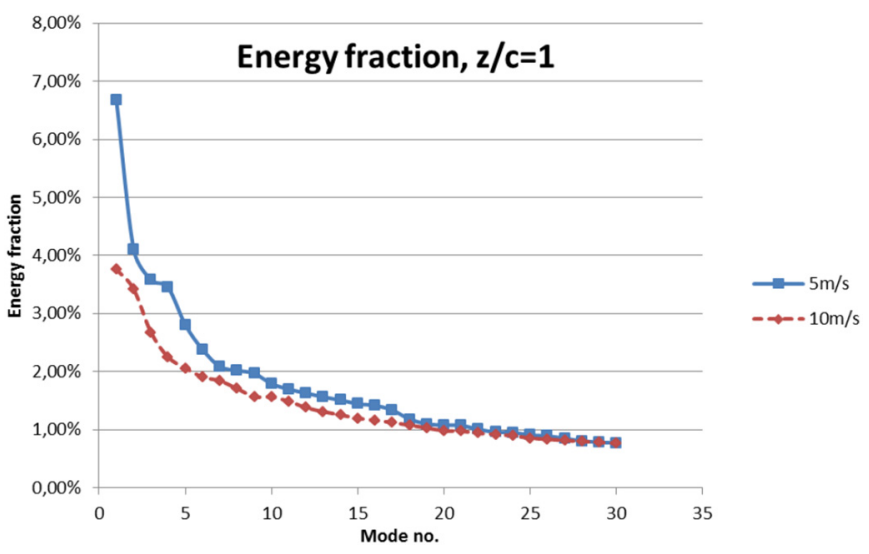

Fig. 11. Energy fraction of first 30 POD modes in plane $z / c=1$ for both examined velocities.

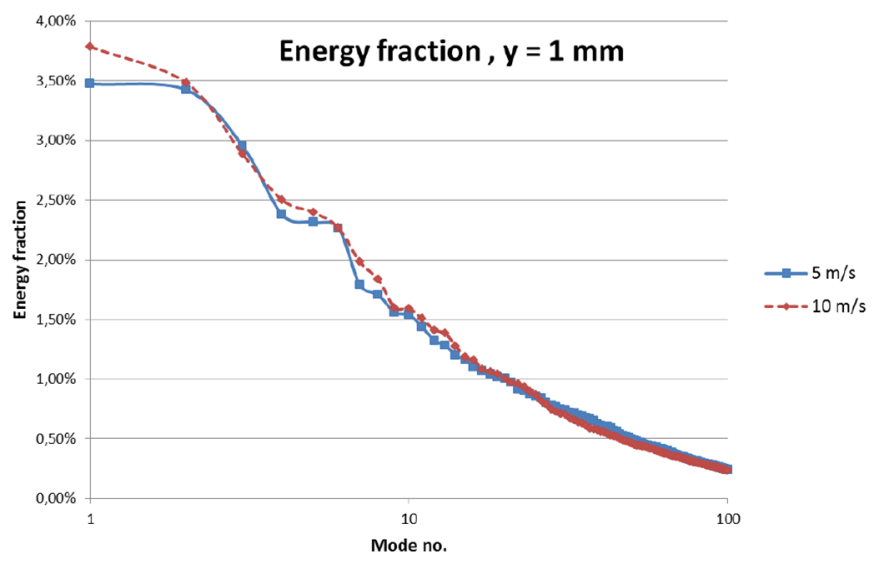

Fig. 12. Energy fraction of first 100 POD modes in parallel plane $y=1 \mathrm{~mm}$ for both examined velocities. 
3. POD mode, $z / c=1.3 \quad 4.4 \%$

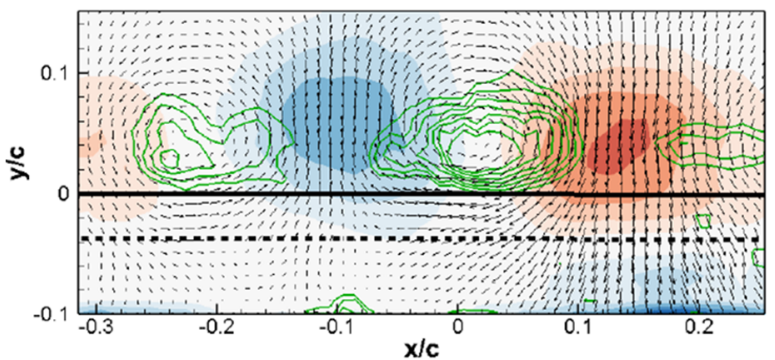

(a)

\section{POD mode, $z / c=1.8 \quad 4 \%$}

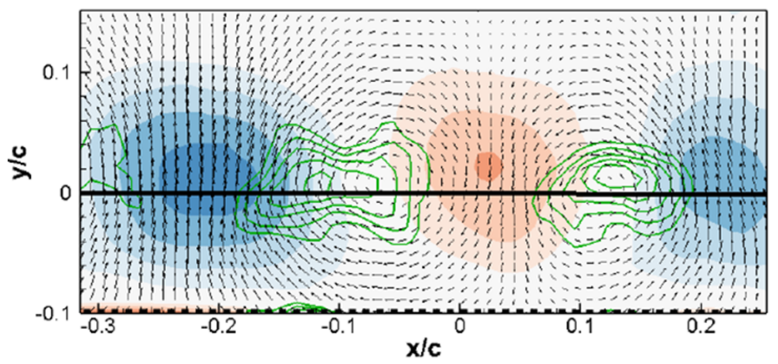

(c)

\section{4. $P O D$ mode, $z / c=2.3 \quad 4.4 \%$}

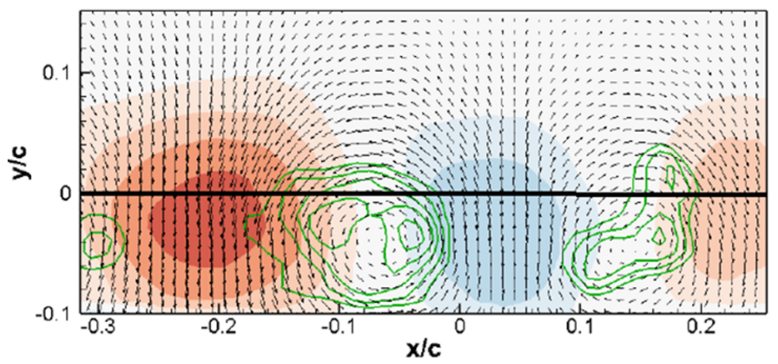

(e)
2. $\mathrm{POD}$ mode, $z / c=1.5 \quad 5.5 \%$

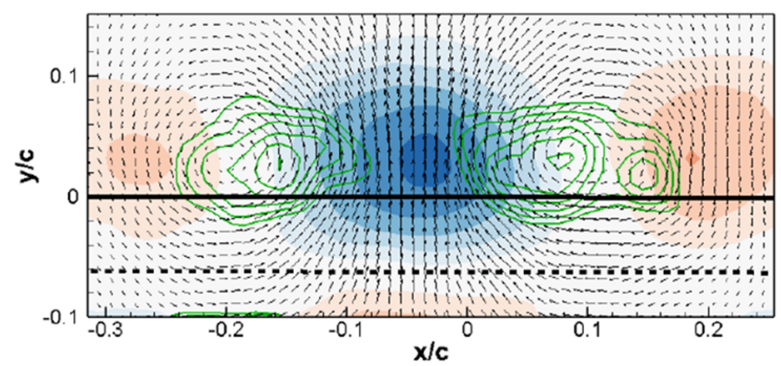

(b)

\section{POD mode, $z / c=2 \quad 4 \%$}

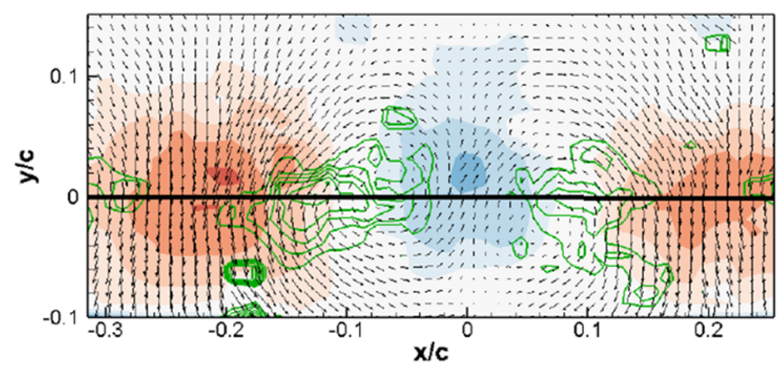

(d)

\section{POD mode, $z / c=2.5 \quad 4.4 \%$}

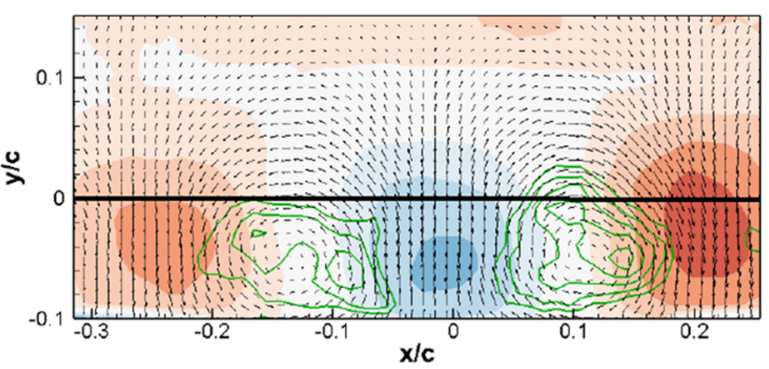

(f)

Fig. 13. POD modes in the form of one pair of vortices in planes $z / c=1.3$ (a), 1.5 (b), 1.8 (c), 2 (d), 2.3 (e) and 2.5 (f), distribution of fluctuation of streamwise velocity, isolines of vorticity, energy portion.

detected for inlet velocity $10 \mathrm{~ms}^{1}$ in the case of parallel plane of measurement. POD method is very sensitive to incorrect vectors. The differences between these two velocities are so small that they can be caused by the inaccuracies. The comparison of mode energy content of several perpendicular planes has revealed that the farthest plane $z / c=2.5$ consists of rather bigger structures created by merging [11].

The topology of each POD mode will be described by inplane vector distribution and by color distribution. The color is depicted for velocity component in streamwise direction (Figs. 13-15). POD calculation is coming out from velocity fluctuation which means that red color is used here for positive velocity differences and hence the region of accelerated velocity and blue color is used for negative differences (region of velocity deceleration). The vorticity in plane of measurement is calculated to detect each individual vortical structure (the shear stresses are not expected). Vorticity isolines are plotted by green solid lines and the vortex is located in their center. Higher density of isolines means more powerful vortex. Isolines do not show the sign of vorticity but all vortices are of alternate motion - clockwise and counter-clockwise.

Dominant coherent structures were detected in the upper part of the wake (above the dashed black line). The vortical structures location is shifting below with increasing distance from the trailing edge. This is the evidence of the downwash effect on the most energetic structures. One of the most important topology is created by one pair of counter-rotating vortices (Fig. 13). This topology was 
7. $\mathrm{POD}$ mode, $\mathrm{z} / \mathrm{c}=1.3 \quad 2.7 \%$

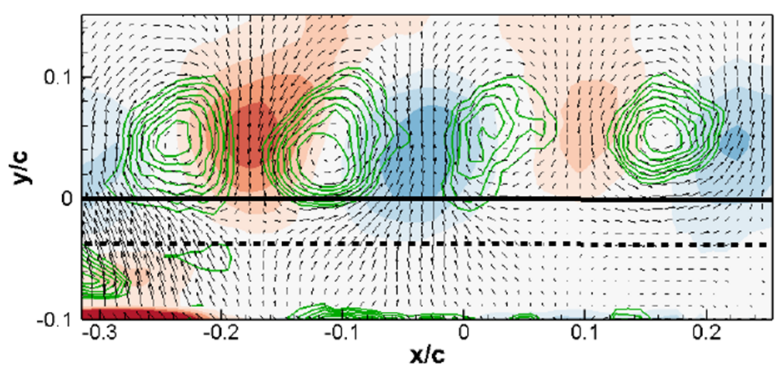

(a)

\section{POD mode, $\mathrm{z} / \mathrm{c}=1.8 \quad 2.5 \%$}

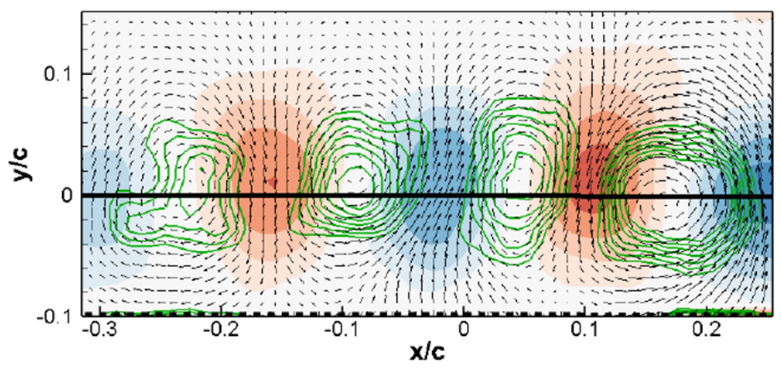

(c)
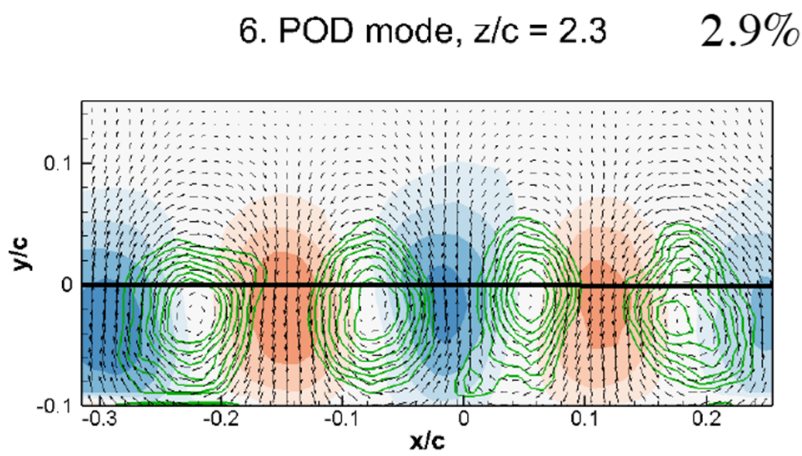

(e)
6. $P O D$ mode, $z / c=1.5 \quad 3 \%$

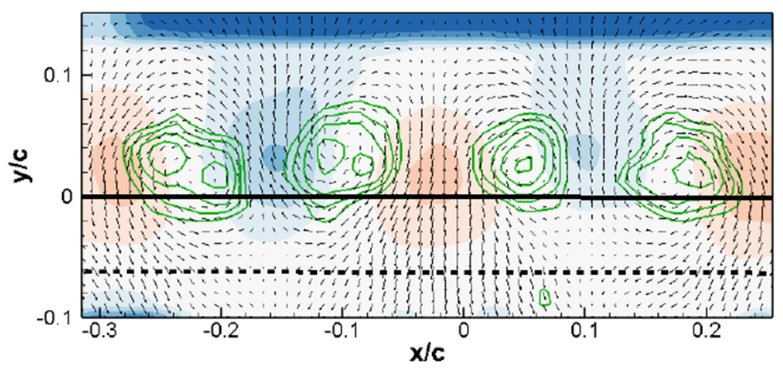

(b)

7. POD mode, $z / c=2$ $2 \%$

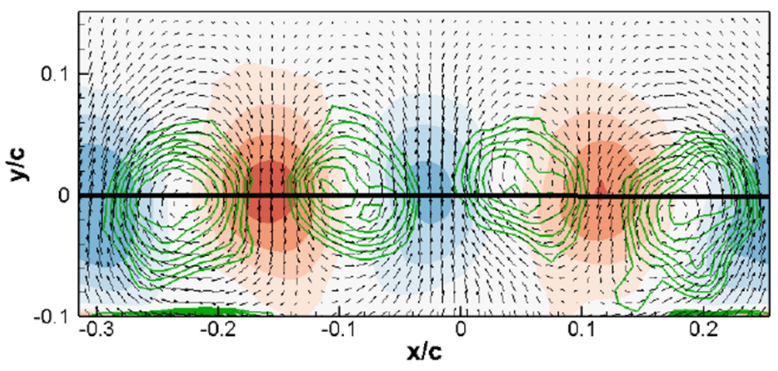

(d)

\section{7. $P O D$ mode, $z / c=2.5 \quad 2.3 \%$}

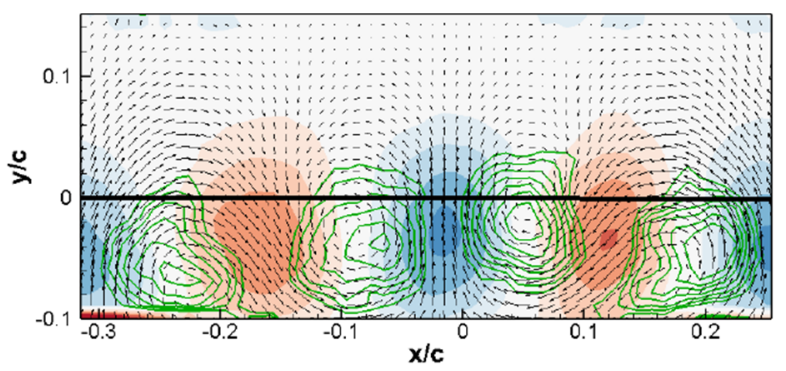

(f)

Fig. 14. POD modes in the form of two pairs of vortices in planes $z / c=1.3$ (a), 1.5 (b), 1.8 (c), 2 (d), 2.3 (e) and 2.5 (f), distribution of fluctuation of streamwise velocity, isolines of vorticity, energy portion.

found for every perpendicular plane $z / c=1.3,1.5,1.8,2$, 2.3 and 2.5 but not for the identical mode number (mode No. alternates between 2 and 5). Nevertheless, this structure dominates for the flow in the whole wake. The space between two vortices is filled with the fluid of either high or low streamwise velocity. The region of streaks as well as the dimension of these coherent structures is not dependent on streamwise direction, only the position of vortices is shifted below. All these modes are associated with kinetic energy of approximately $4.5 \%$.

Similar flow pattern were detected from modes No. 6 and 7 (energy contain about 2.5\%) - here the mode topology is created by two pairs of counter-rotating vortices with space between them filed by accelerated or decelerated fluid (Fig. 14). Except the downwash, there is again no dependency on $z$-coordinate. The size of vortices is significantly smaller compared with previous ones. This is why they contain half amount of kinetic energy. Highorder modes are more structured and complex and contain tiny amount of energy. The example is given in Figure 15. Still, it is valid that structures are located above dashed black line (the projection in the direction of plate inclination).

POD decomposition of flow field measured in parallel plane was based mainly on out-of-plane velocity component $v$ (which is denoted by color). $\mathrm{U}$ and $\mathrm{w}$-component is represented by black vectors. The most energetic POD modes is the first one and second one (both contain about 


\section{3. $P O D$ mode, $z / c=1.5 \quad 1.6 \%$}

14. POD mode, $z / c=1.8$

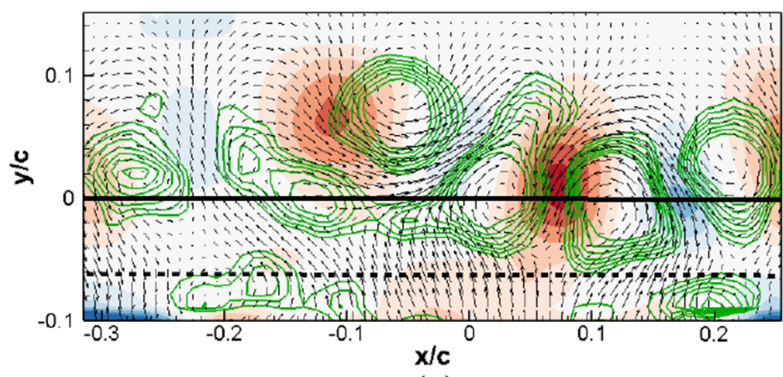

(a)

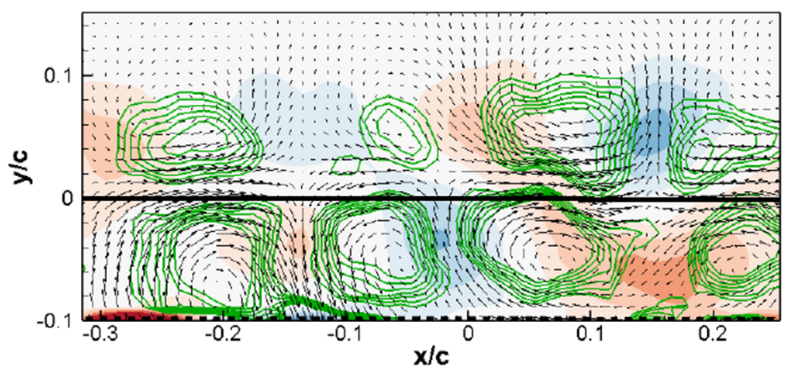

(b)

Fig. 15. POD modes in the form of complex vorticity field in planes $z / c=1.5$ (a) and 1.8 (b), distribution of fluctuation of streamwise velocity, isolines of vorticity, energy portion.

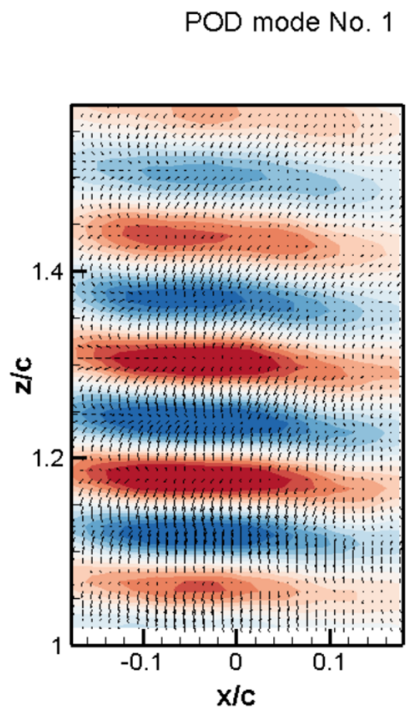

(a)

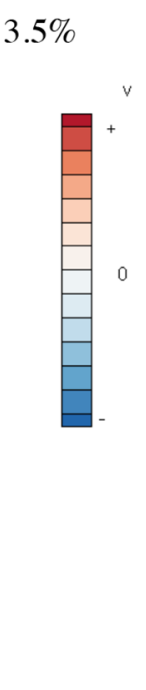

Fig. 16. 1st (a) and 2nd (b) POD mode topology, distribution of v-component of velocity and the energy portion.
$3.5 \%$ ) which is characterized by the train of spanwise oriented vortices spread along the span dimension so that there is no change along $x$-coordinate (Fig. 16). Both modes differ only by a phase shift. These modes could represent the vortex shedding at the trailing edge.

There are other modes with lower energy content calculated in the same plane in Figure 17. The third POD mode contains about 3\% of energy and is represented by synchronous pulsations of the in-plane velocity components. Similar modes were also detected in the other position of dataset. Typical chequered pattern of vcomponent of velocity is captured by mode No. 4, 9 and 20. There is one change in the mode No. 4 , two changes in the mode No. 9 and three changes in the mode No. 20 with respect to analyzed range of $x$-coordinate. On the other hand, the distribution of the opposite $v$ velocity component orientation in streamwise coordinate is quite similar to the first two modes which implies that they also represent the moving train of vortices. Figure 18 is plotted here to shown the high-order modes and their complexity. Their energy is smaller than $1 \%$ and their role seems to be very low although they consist of structures which have oblique orientation. Similar structures were detected by Schlatter [12] in DNS simulation around an airfoil, although the Reynolds number was significantly bigger in the case of simulation.

The last Figure 19 is plotted here to show the oblique modes detected for inlet velocity $10 \mathrm{~m} \mathrm{~s}^{1}$.

\section{Conclusion and discussion}

This study presents results supporting the new theory of flight explaining origin of lift force on an airfoil by 
POD mode No. 3

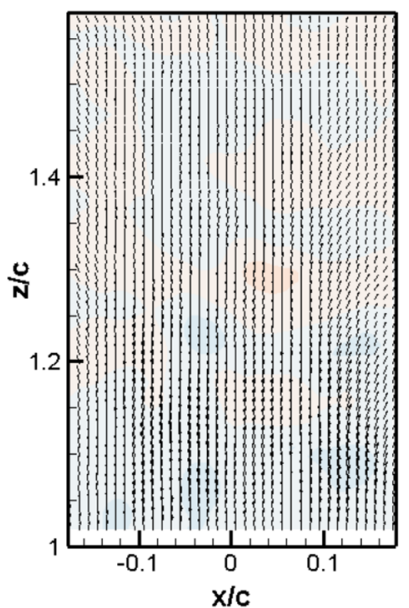

(a)

POD mode No. 9

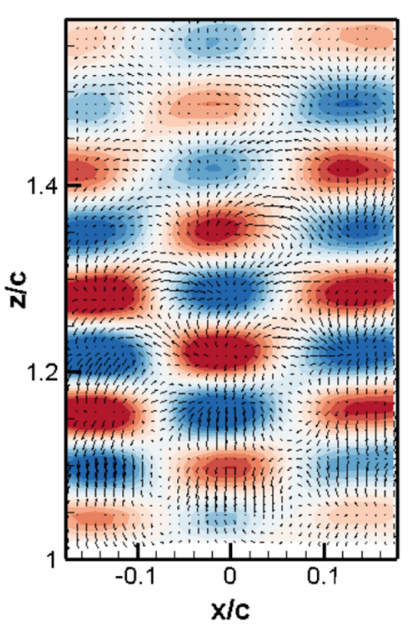

(c)
$3 \%$

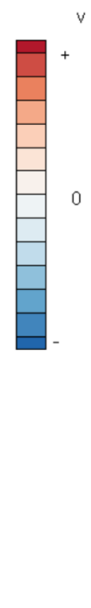

$1.6 \%$

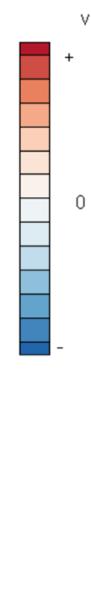

Fig. 17. Examples of chequered modes (b, c, d) and a mode of synchronous pulsations (a).

presence of streamwise fluctuating vorticity. The detailed experimental research of the unsteady flow around an inclined flat plate was carried out. Instantaneous velocity and vorticity fields have been shown in selected measuring planes, describing the dynamical topology of the flow-field.

The flow topology approaches 2D structure for statistical quantities only, streamwise vorticity is not present in the mean flow. The instantaneous flow field is fully turbulent and 3D with streamwise and spanwiseoriented vortical structures subjected to merging. Rotational slip separation was studied close to the trailing edge and inside the wake. The downwash effect was demonstrated by streamlines, instantaneous vorticity as well as position of dominant coherence structure in POD modes.
POD mode No. 4

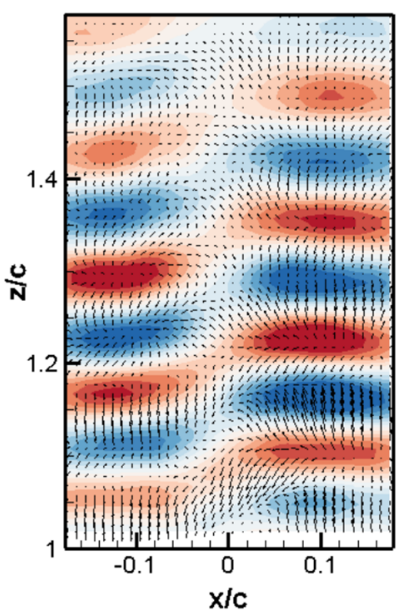

(b)

POD mode No. 20

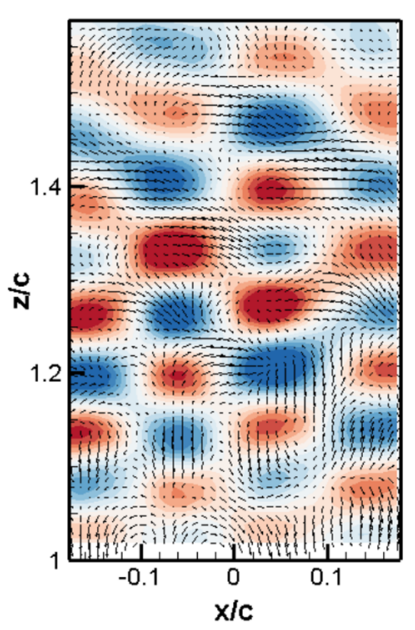

(d)
$2.4 \%$

$1 \%$

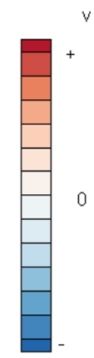

The POD decomposition has detected the streamwise vortical structures in the perpendicular plane of measurement. The origin of these vortices is located at the suction side of the plate. The plate pressure side is absolutely poor in such structures and that is why they are detected rather in the upper part of the wake.

The measurement in the plane parallel to the airfoil has shown that the wake is filed by spanwise oriented (and also oblique) vortices. These can be decomposed into chequered patterns.

The results of presented experiments fully support the new theory of flight and lift generation.

However, the study is of qualitative nature showing possible flow topologies preferably. Estimation of an individual structure contribution is not possible, as pressure distribution is not evaluated in the presented study. 


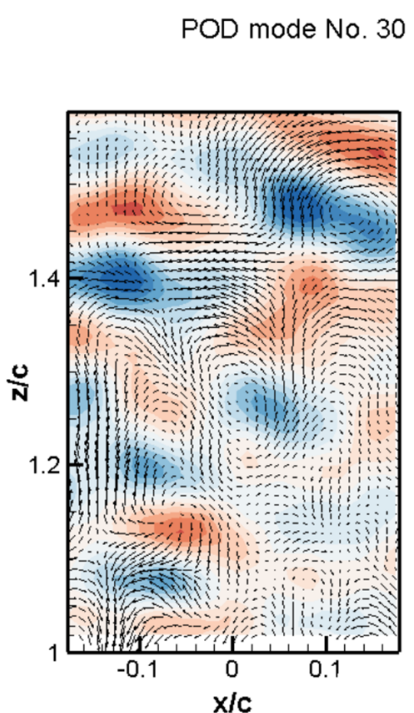

(a)
$0.7 \%$

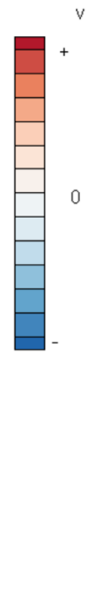

Fig. 18. Examples of oblique (a, b) and higher (b) POD modes.

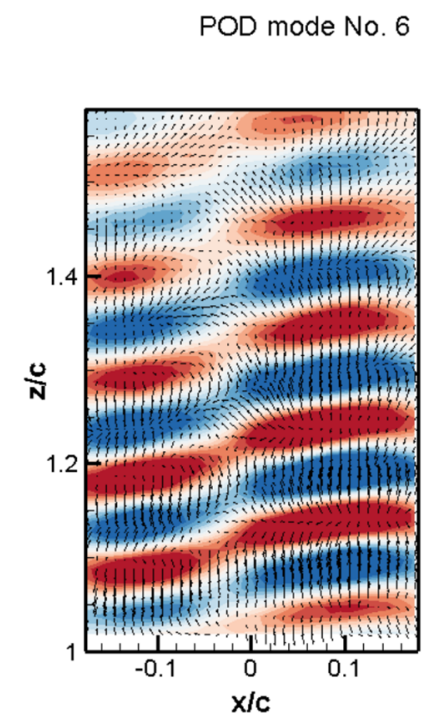

(a)

\section{$2.3 \%$}

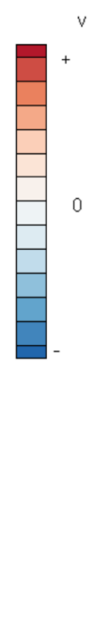

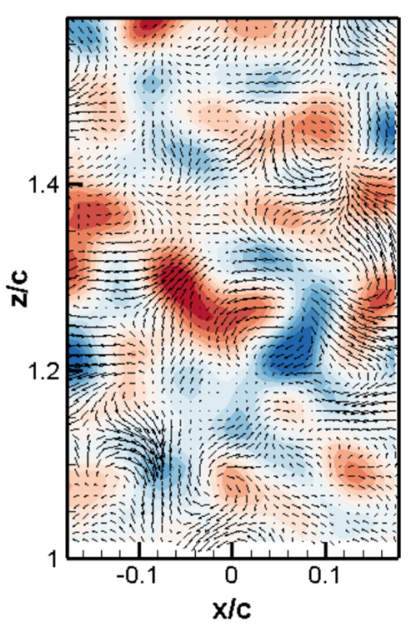

(b)

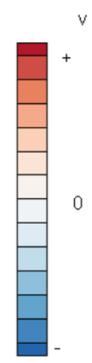

Fig. 19. Examples of POD modes acquired for freestream velocity $10 \mathrm{~m} \mathrm{~s}^{-1}$.

\section{Nomenclature}

$x, y, z \quad$ Cartesian coordinates $[\mathrm{m}]$

$u, v, w \quad$ Velocity components $\left[\mathrm{m} \mathrm{s}^{-1}\right]$

$u^{\prime}, v^{\prime}, w^{\prime} \quad$ Fluctuation of velocity components $\left[\mathrm{m} \mathrm{s}^{-1}\right]$

$c \quad$ Chord length $[\mathrm{m}]$

$\omega \quad$ Vorticity $\left[\mathrm{s}^{-1}\right]$

TKE Turbulent Kinetic Energy $\left[\mathrm{m}^{2} \mathrm{~s}^{-2}\right]$

PIV Particle Image Velocimetry

POD Proper Orthogonal Decomposition

The authors gratefully acknowledge the financial support of the Grant Agency of the Czech Republic, project No. 17-01088S.

\section{References}

1 R. von Mises, Theory of Flight, McGraw-Hill, New York, 1945

2 H. Schlichting, Boundary Layer Theory, McGraw-Hill, New York, 1979

3 J.D. Anderson, A history of aerodynamics, Cambridge Aerospace Series 8, Cambridge University Press, Cambridge, 1997

4 E.L. Houghton, P.W. Carpenter, Aerodynamics for Engineering Students, Elsevier, Amsterdam, 2003

5 J.D. Anderson, Fundamentals of Aerodynamics, McGrawHill Education, New York, 2016

6 J. Hoffman, J. Jansson, C. Johnson, New theory of flight, J. Math. Fluid Mech. 18, 219-241 (2016) 
7 J. Hoffman, C. Johnson, Resolution of d'Alambert's paradox, J. Math. Fluid Mech. 12, 321-334 (2010)

8 V. Uruba, P. Procházka, On the global flow-field dynamics around an airfoil, EPJ Web Conf., to be published (2019)

9 V. Uruba, P. Procházka, V. Skála, On the structure of the boundary layer under adverse pressure gradient on an inclined plate, J. Phys. Conf. Ser. 1101, 012047 (2018)
10 V. Uruba, Decomposition methods in turbulent research, EPJ Web Conf. 25, 01095 (2012)

11 P. Procházka, V. Uruba, V. Skála, Evolution of vortical structures behind an inclined flat plate, Matec Web Conf. 168, 05003 (2018)

12 P. Schlatter, Q. Li, R. Örlü, F. Hussain, D.S. Henningson, On the near-wall vortical structures at moderate Reynolds numbers, Eur. J. Mech. B/Fluids 48, 75-93 (2014)

Cite this article as: P. Procházka, V. Uruba, Streamwise and spanwise vortical structure merging inside the wake of an inclined flat plate, Mechanics \& Industry 20, 705 (2019) 